\title{
A REMARK ON THE SUM AND THE INTERSECTION OF TWO NORMAL IDEALS IN AN ALGEBRA
}

\author{
TADASI NAKAYAMA
}

Let $F$ be a quotient field of a commutative domain of integrity $o$ in which the usual arithmetic holds. ${ }^{1}$ Consider an algebra $\mathfrak{A}$ with a unit element over $F$. Let $\Im_{1}, \Im_{2}, \Im_{3}, \Im_{4}$ be four arbitrary maximal orders in $\mathfrak{A}$ and $\mathfrak{a}, \mathfrak{b}, \mathfrak{c}$ be three arbitrary normal ideals. We prove the following theorems.

THEOREM 1. If $\Im_{1} \cap \Im_{2}=\Im_{3} \cap \Im_{4}\left[\right.$ or $\left.\left(\Im_{1}, \Im_{2}\right)=\left(\Im_{3}, \Im_{4}\right)\right]$, then either $\Im_{1}=\Im_{3}, \Im_{2}=\Im_{4}$ or $\Im_{1}=\Im_{4}, \Im_{2}=\Im_{3}$.

THEOREM 2. Both the left and the right orders of $\left(\Im_{1}, \Im_{2}\right)$ are $\Im_{1} \cap \Im_{2}$. Also $\Im_{1} \cap \Im_{2} \subseteq \Im_{3}$ if and only if $\left(\Im_{1}, \Im_{2}\right) \supseteqq \Im_{3}$; if this is the case the dis-

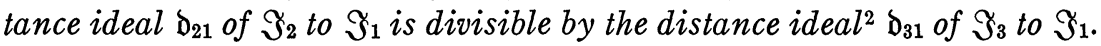

THEOREM 3. The left, say, order $\mathfrak{b}$ of the intersection $\mathfrak{a} \cap \mathfrak{b}$ [the sum $(\mathfrak{a}, \mathfrak{b})]$ is an intersection of two suitable maximal orders.

More precisely, if $\mathfrak{r}$ and $\mathbb{z}$ are normal ideals such that $\mathfrak{b}=\mathfrak{r a z}$ in the sense of proper multiplication and if $t$ is the smallest two-sided ideal of the right order of $\mathfrak{a}$ which divides $\mathbb{z}$ while $\mathrm{t}^{\prime}$ is the largest twosided ideal of the same maximal order which is divisible by $\mathbb{z}$, then $\mathfrak{D}$ is the intersection of the left orders of the two normal ideals $a \mathbf{n} \mathfrak{r a t}$ and $\mathfrak{a} \boldsymbol{n} \mathfrak{r a t} \mathfrak{t}^{\prime}\left[(\mathfrak{a}, \mathfrak{r a t})\right.$ and $\left.\left(\mathfrak{a}, \mathfrak{r a t} \mathfrak{t}^{\prime}\right)\right] \cdot{ }^{3}$ The left order of $\mathfrak{a} \mathbf{n} \mathfrak{b}$ coincides with the right order of $\left(\mathfrak{a}^{-1}, \mathfrak{b}^{-1}\right)$.

THEOREM 4. $\mathfrak{a} \cap \mathfrak{b} \leqq \mathfrak{c}$ implies $\left(\mathfrak{a}^{-1}, \mathfrak{b}^{-1}\right) \supseteqq \mathfrak{c}^{-1}$ and conversely.

For the proof we have, according to the well known reduction, only to treat the case where $F$ is a $p$-adic field $F=F_{p}$ and $\mathfrak{A}$ is a normal simple algebra over $F$. Then $\mathfrak{A}$ is a (complete) matric ring $D_{r}=\sum_{i, k=1}^{r} \epsilon_{i k} D$ over a division algebra $D$, where $\epsilon_{i k}$ is a system of matric units commutative with every element of $D$. D possesses a unique maximal order $I$, and $I$ has a unique prime ideal $P$.

Notation. If $a_{i k},(i, k=1,2, \cdots, r)$, is a system of rational integers, we denote by $M\left(a_{i k}\right)$ the ideal $\sum_{i, k} \epsilon_{i k} P^{a i k}$ in $\mathfrak{A}$.

\footnotetext{
${ }^{1}$ In the following we shall adopt the terminologies used in M. Deuring, Algebren, Ergebnisse der Mathematik, vol. 4, no. 1, 1935.

2 If the algebra is a quaternion algebra, then the converse is also valid. Cf. M. Eichler, Journal für die reine und angewandte Mathematik, vol. 174 (1936), §7.

${ }^{3}$ Thus the intersection and the sum are no more normal ideals except for trivial cases; cf. Nakayama, Proceedings of the Imperial Academy of Japan, vol. 12 (1936).
} 
$M\left(a_{i k}\right)$ is an order if and only if $a_{i i}=0, a_{i l}+a_{l k} \geqq a_{i k}$ for all $i, k, l$. On assuming this condition it is a maximal order if and only if $\sum a_{i k}=0$. By a simple calculation we then have the following lemma. ${ }^{4}$

Lemma 1. A necessary and sufficient condition that $M\left(a_{i k}\right)$ be a maximal order is that there should exist $r$ rational integers $c_{i}$ such that $a_{i k}=c_{k}-c_{i}$. Every normal ideal whose left and right orders are $M\left(c_{k}-c_{i}\right)$ and $M\left(d_{k}-d_{i}\right)$ respectively has the form $P^{a} M\left(d_{k}-c_{i}\right)=M\left(d_{k}-c_{i}+a\right)$.

It follows from a lemma of Chevalley ${ }^{5}$ that a maximal order in $\mathfrak{A}$ has really the form $M\left(a_{i k}\right)$ (whence the form $M\left(c_{k}-c_{i}\right)$ ) whenever it contains all diagonal $\epsilon_{11}, \epsilon_{22}, \cdots, \epsilon_{r r}$.

Lemma 2. There exists a regular element $\alpha$ in $\mathfrak{A}$ such that

$$
\alpha^{-1} \Im_{1} \alpha=M(0), \quad \alpha^{-1} \mathfrak{\Im}_{2} \alpha=M\left(c_{k}-c_{i}\right) ; \quad c_{1} \geqq c_{2} \geqq \cdots \geqq c_{r} .
$$

Proof. There is, as is well known, a regular element $\beta$ such that $\beta^{-1} \Im_{1} \beta=M(0)$. Consider the distance ideal $\delta_{12}=\left(\Im_{2} \Im_{1}\right)^{-1}=\Im_{1} \delta$ of $\Im_{1}$ to $\Im_{2}$. The theory of elementary divisors tells the existence of two units $\xi, \eta$ in $M(0)$ such that $\gamma=\xi \beta^{-1} \delta \beta \eta$ is a diagonal matrix with diagonal elements $P^{c i},\left(c_{1} \geqq \cdots \geqq c_{r}\right), \gamma=\sum \epsilon_{i i} P^{c i}$, where we denote, for the sake of convenience, a prime element of the prime ideal $P$ by the same letter $P$. Put $\alpha=\beta \eta$. Then this $\alpha$ possesses the required property: $\alpha^{-1} \Im_{1} \alpha=\eta^{-1} \beta^{-1} \Im_{1} \beta \eta=M(0), \alpha^{-1} \Im_{2} \alpha=\gamma^{-1} M(0) \gamma=M\left(c_{k}-c_{i}\right)$.

Lemma 3. There exist two regular elements $\alpha, \beta$ in $\mathfrak{A}$ such that

$$
\begin{aligned}
& \alpha \mathfrak{a} \beta=M(0), \quad \alpha \mathfrak{b} \beta=M\left(d_{k}-c_{i}\right) ; \\
& c_{1} \geqq c_{2} \geqq \cdots \geqq c_{r}, d_{1} \geqq d_{2} \geqq \cdots \geqq d_{r} .
\end{aligned}
$$

Proof. Let $\Im_{1}^{\prime}, \Im_{2}^{\prime}\left[\Im_{3}^{\prime}, \Im_{4}^{\prime}\right]$ be the left and the right orders of $\mathfrak{a}$ $[\mathfrak{b}]$. According to the above lemma there exist $\gamma, \beta$ such that $\gamma^{-1} \Im_{1}^{\prime} \gamma=\beta^{-1} \Im_{2}^{\prime} \beta=M(0), \gamma^{-1} \Im_{3}^{\prime} \gamma=M\left(c_{k}-c_{i}\right), \beta^{-1} \Im_{4}^{\prime} \beta=M\left(d_{k}^{\prime}-d_{i}^{\prime}\right)$. $\gamma^{-1} \mathfrak{a} \beta$ is a two-sided ideal of $M(0)$ and has a form $P^{a} M(0)$. Put $\alpha=\left(\gamma P^{a}\right)^{-1}$. Then $\alpha \mathfrak{a} \beta=M(0)$. Moreover, $\alpha \mathfrak{b} \beta$ is of a form $M\left(d_{k}^{\prime}-c_{i}+b\right)$ (Lemma 1). We put $d_{k}=d_{k}^{\prime}+b$, and this completes the proof.

We note further that the left order of an ideal $M\left(a_{i k}\right)$ is $M\left(b_{i k}\right)$ where $b_{i k}=\max _{j}\left(a_{i j}-a_{k j}\right)$.

After these preliminaries our theorems are easy to prove. In Theorem 1 we may, according to Lemma 2 , assume that $\Im_{1}=M(0)$, $\Im_{2}=M\left(c_{k}-c_{i}\right), \quad\left(c_{1} \geqq \cdots \geqq c_{r}\right)$. Suppose $\Im_{1} \cap \Im_{2}=\Im_{3} \cap \Im_{4}$. Since

${ }^{4}$ Cf. Nakayama, Japanese Journal of Mathematics, vol. 13 (1937), p. 339.

${ }^{5}$ Chevalley, Abhandlungen aus dem mathematischen Seminar der Hamburgischen Universität, vol. 10 (1934), p. 87. 
$\epsilon_{i i} \varepsilon \Im_{1} \cap \Im_{2} \cong \Im_{3}, \Im_{4}$, it follows that $\Im_{3}, \Im_{4}$ have the form $\Im_{3}=M\left(d_{k}-d_{i}\right)$, $\Im_{4}=M\left(f_{k}-f_{i}\right)$. Moreover $\max \left(d_{k}-d_{i}, f_{k}-f_{i}\right)=\max \left(0, c_{k}-c_{i}\right)$, $(i, k=1,2, \cdots, r)$. This implies $\max \left(d_{k}-d_{i}, f_{k}-f_{i}\right)=0$ if $i \geqq k$, whence $d_{1} \geqq \cdots \geqq d_{r}$ and $f_{1} \geqq \cdots \geqq f_{r}$. On applying the same relation to $i=1, k=r$, we find that either $d_{1}=d_{r}$ or $f_{1}=f_{r}$. In the first case we have $d_{1}=\cdots=d_{r}, f_{1}-f_{i}=c_{1}-c_{i}, \quad(i=1,2, \cdots, r)$, whence $\Im_{1}=\Im_{3}, \Im_{2}=\Im_{4}$. The second case gives of course $\Im_{1}=\Im_{4}, \Im_{2}=\Im_{3}$.

The assertion about the sum follows now from Theorem 2, which is in turn contained in Theorems 3 and 4 .

As to Theorem 3 we notice first that if $\alpha, \beta$ are two regular elements, the ideals $\alpha \mathrm{r} \alpha^{-1}, \beta^{-1} \mathbb{B} \beta, \beta^{-1} \mathrm{t} \beta, \beta^{-1} \mathrm{t}^{\prime} \beta$ have the same significance for $\alpha \mathfrak{a} \beta$ and $\alpha \mathfrak{b} \beta$ as the ideals $\mathfrak{r}, \mathfrak{b}, \mathfrak{t}, \mathfrak{t}^{\prime}$ have for $\mathfrak{a}$ and $\mathfrak{b}$. Hence it is sufficient, by Lemma 3, to consider the case where

$$
\begin{aligned}
& \mathfrak{a}=M(0), \quad \mathfrak{b}=M\left(d_{k}-c_{i}\right) ; \\
& c_{1} \geqq c_{2} \geqq \cdots \geqq c_{r}, d_{1} \geqq d_{2} \geqq \cdots \geqq d_{r} .
\end{aligned}
$$

Then $\mathfrak{a} \cap \mathfrak{b}=M\left(\max \left(0, d_{k}-c_{i}\right)\right)$ and $\mathfrak{D}=M\left(a_{i k}\right)$ with

$$
\begin{aligned}
a_{i k} & =\max _{j}\left(\max \left(0, d_{j}-c_{i}\right)-\max \left(0, d_{i}-c_{k}\right)\right) \\
& =\left\{\begin{array}{l}
\max \left(0, d_{1}-c_{i}\right)-\max \left(0, d_{1}-c_{k}\right)=f_{k}-f_{i} \text { for } i \geqq k, \\
\max \left(0, d_{r}-c_{i}\right)-\max \left(0, d_{r}-c_{i}\right)=g_{k}-g_{i} \text { for } i \leqq k,
\end{array}\right.
\end{aligned}
$$

where $f_{i}=-\max \left(0, d_{1}-c_{i}\right), g_{i}=-\max \left(0, d_{r}-c_{i}\right)$. Since $f_{k}-f_{i} \geqq$ or $\leqq g_{k}-g_{i}$ according as $i \geqq k$ or $i \leqq k$, we find that $\mathrm{D}$ is the intersection of the two maximal orders $M\left(f_{k}-f_{i}\right)$ and $M\left(g_{k}-g_{i}\right)$. Further, if we put $\gamma=\sum \epsilon_{i i} P^{-c_{i}}, \delta=\sum \epsilon_{i i} P^{-d_{i}}$, then $\mathfrak{r}=\gamma P^{a} M(0)$ and $\mathfrak{z}=M(0) P^{-a} \delta$, whence $\mathrm{t}=P^{a-d_{r}} M(0), \mathrm{t}^{\prime}=P^{d_{1}-a} M(0)$. From this we can easily verify the precise characterization of 0 given in the theorem.

The part on the sum $(\mathfrak{a}, \mathfrak{b})$ can be shown by a similar computation. And indeed from that computation we obtain the last assertion in the theorem.

Finally, to prove Theorem 4 we observe again that we have only to consider the case where $\mathfrak{a}, \mathfrak{b}$ have the form (1). $\mathfrak{a} \mathbf{n} \mathfrak{b}=M(\max (0$, $\left.d_{k}-c_{i}\right),\left(\mathfrak{a}^{-1}, \mathfrak{b}^{-1}\right)=M\left(\min \left(0, c_{k}-d_{i}\right)\right)$ because $\mathfrak{b}^{-1}=M\left(c_{k}-d_{i}\right)$, and here we notice that $\max \left(0, d_{k}-c_{i}\right)=-\min \left(0, c_{i}-d_{k}\right)$. The third normal ideal $\mathfrak{c}$ can be expressed as $\mathfrak{c}=\tau^{-1} M(0) \sigma^{-1}$ with regular elements $\sigma=\sum \epsilon_{i k} s_{i k}, \tau=\sum \epsilon_{i k} t_{i k}$. Let $P^{c i k}$ be the exact power of $P$ which divides $s_{i k}, P^{c_{i k}} \| s_{i k}$; if $s_{i k}=0$ we put $c_{i k}=\infty$. Let similarly $P^{d_{i k}} \| t_{i k}$. It is evident that $M\left(a_{i k}\right)$, with a system of rational integers $a_{i k}$, contains $\mathfrak{c}^{-1}=\sigma M(0) \tau$ if and only if

$$
c_{i j}+d_{l k} \geqq a_{i k}, \quad \text { for all } i, j, k, l .
$$


Hence, if we show that the same condition is also necessary and sufficient in order that $M\left(-a_{k i}\right) \leqq \mathfrak{c}$, then we will be through. But this is also easy to see. For, $\mathfrak{c}=\tau^{-1} M(0) \sigma^{-1}$ consists of all $\eta=\sum \epsilon_{i k} y_{i k}$ $=\tau^{-1}\left(\sum \epsilon_{i k} x_{i k}\right) \sigma^{-1}$ with $x_{i k} \varepsilon I$. On taking a pair $(j, l)$ of indices, let us consider those $\eta$ such that $y_{i k}=0$ for $(i, k) \neq(j, l)$. In other words, we consider the equation $\tau^{-1}\left(\sum \epsilon_{i k} x_{i k}\right) \sigma^{-1}=\epsilon_{j l} y_{j l}$. But this is equivalent to $\sum \epsilon_{i k} x_{i k}=\tau \epsilon_{j l} y_{j l} \sigma$, or

$$
x_{i k}=t_{i j} y_{j l} s_{l k}, \quad i, k=1,2, \cdots, r .
$$

Suppose now $M\left(-a_{k i}\right) \subseteq c$. Then (3) with $y_{j l}=P^{-a_{l j}}$ must have a solution $x_{i k} \varepsilon I$. Hence $0 \leqq d_{i j}-a_{l j}+c_{l k}$ (for all $\left.i, k\right)$. Since $(j, l)$ was an arbitrary pair of indices, we have thus established (2). Assume conversely (2). Then obviously $x_{i k}=t_{i j} P^{-a_{l i} s_{l k} \varepsilon} I$ whence $\epsilon_{j l} P^{-a_{l j} \varepsilon \mathfrak{c} \text { and }}$ $M\left(-a_{k i}\right) \subseteq \mathrm{c}$.

A second proof of the last part of Theorem 3 is as follows: We observe first that every ideal $\mathfrak{m}$ in $\mathfrak{A}$ is additively generated by regular elements contained in $\mathrm{m}^{6}{ }^{6}$ For, if $\xi \varepsilon \mathfrak{m}$ we take a scalar element $a(\varepsilon F)$ in $\mathfrak{m}$ different from all the characteristic roots of the matrix which represents $\xi$ in a faithful representation of $\mathfrak{A}$. Then $\xi-a(\varepsilon \mathfrak{m})$ is evidently a regular element and $\xi=(\xi-a)+a$. Now, let $\alpha$ be any regular element from the left order of $\mathfrak{a} \cap \mathfrak{b} ; \alpha(\mathfrak{a} \cap \mathfrak{b}) \leqq \mathfrak{a} \mathbf{n} \mathfrak{b}$. Since $\alpha \mathfrak{a}$ and $\alpha \mathfrak{b}$ are normal ideals, we have, from Theorem $4,\left(\mathfrak{a}^{-1} \alpha^{-1}, \mathfrak{b}^{-1} \alpha^{-1}\right) \supseteqq \mathfrak{a}^{-1}$, $\mathfrak{b}^{-1}$ whence $\left(\mathfrak{a}^{-1}, \mathfrak{b}^{-1}\right) \alpha^{-1} \supseteqq\left(\mathfrak{a}^{-1}, \mathfrak{b}^{-1}\right),\left(\mathfrak{a}^{-1}, \mathfrak{b}^{-1}\right) \supseteqq\left(\mathfrak{a}^{-1}, \mathfrak{b}^{-1}\right) \alpha$. This shows that the left order of $\mathfrak{a} \mathbf{n} \mathfrak{b}$ is contained in the right order of $\left(\mathfrak{a}^{-1}, \mathfrak{b}^{-1}\right)$. But the converse can be seen in quite a similar manner.

Remark. The structure of the residue class algebra $\Im_{1} \cap \Im_{2} / p\left(\Im_{1} \cap \Im_{2}\right)$ is easy to analyze, but perhaps does not deserve a detailed discussion. We merely note that the algebra is not symmetric, in fact is not weakly symmetric, ${ }^{7}$ except for the trivial case $\left(\Im_{1}\right)_{p}=\left(\Im_{2}\right)_{p}$; this remark may be of some interest in view of a recent paper by R. Brauer. ${ }^{8}$

The Institute for Advanced Study

${ }^{6}$ We exclude here the trivial case of a finite underlying field $F$.

${ }^{7}$ See Brauer-Nesbitt, Proceedings of the National Academy of Sciences, vol. 23 (1937); Nakayama-Nesbitt, Annals of Mathematics, (2), vol. 39 (1938).

${ }^{8}$ Brauer, On modular and p-adic representations of algebras, Proceedings of the National Academy of Sciences, vol. 25 (1939). 